\title{
Delayed massive hemorrhage due to external iliac artery pseudo- aneurysm and uretero-iliac artery fistula following robotic radical cystectomy and intracorporeal Studer pouch reconstruction: Endovascular management of an unusual complication
}

\author{
Ali Fuat Atmaca, MD, ${ }^{*+}$ Abdullah Erdem Canda, MD, ${ }^{*+}$ Mehmet Gumus, MD, ${ }^{\xi}$ Erem Asil, MD; ${ }^{\dagger}$ \\ Mevlana Derya Balbay, MD*
}

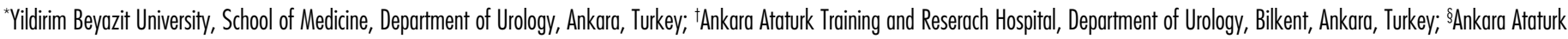
Training and Reserach Hospital, Department of Radiology, Bilkent, Ankara, Turkey; ${ }^{*}$ Memorial Sisli Hospital, Department of Urology, Istanbul, Turkey

Cite as: Can Urol Assoc J 2013;7(9-10):e605-8. http://dx.doi.org/10.5489/cuaj.170 Published online September 10, 2013.

\section{Abstract}

We report a very unusual complication of uretero-iliac artery fistula that developed following robotic radical cystectomy (RARC), bilateral extended pelvic lymph node dissection and intracorporeal Studer pouch reconstruction. Our patient was a 54-year-old male who was admitted 1 month after undergoing robotic surgery due to intermittently occurring massive transurethral bleeding necessitating blood transfusion that stopped by itself. Angiography showed a right external iliac artery pseudo-aneurysm and a fistula tract between the pseudo-aneurysm and Wallace type ureteral anostomosis that was successfully treated by an angiographic endovascular stent insertion at this level. Uretero-iliac artery fistula might occur following RARC, bilateral extended pelvic lymph node dissection and intracorporeal Studer pouch reconstruction leading to intermittently massive transurethral bleeding. Angiography and stenting are important for diagnosis and successful treatment of this rare entity.

\section{Introduction}

Robotic radical cystectomy (RARC) is increasingly being performed worldwide for bladder cancer. In most of the reported series, urinary diversion is performed extracorporeally and the number of centres performing a totally intracorporeal RARC, including urinary diversion and ureteral anastomosis, is very limited. Certainly, this complex robotic surgical procedure is not complication-free. Recently, we published our initial results and surgical techniques in detail related with RARC, bilateral extended pelvic lymph node dissection and intracorporeal Studer pouch reconstruction. ${ }^{1-4}$
We report a very unusual complication of uretero-iliac artery fistula and serious hemorrhage that developed postoperatively following RARC, bilateral extended pelvic lymph node dissection and intracorporeal Studer pouch reconstruction.

\section{Case report}

A 54-year-old male patient underwent RARC, bilateral extended pelvic lymph node dissection and intracorporeal Studer pouch reconstruction for invasive bladder cancer at our institution. He had a cigarette smoking history of 1 package/day for 20 years. Estimated blood loss was 200 cc with console time, including the whole procedure, of 7.5 hours. Postoperative follow-up was uneventful and the patient was discharged on postoperative day 11 . Postoperative pathology revealed pT2N0 disease with negative surgical margins and a lymph node yield of 20 nodes. On follow-up after 1 month, he was admitted to our department due to intermittently occurring massive transurethral bleeding leading to hypotension and a significant drop in blood hemoglobin level necessitating blood transfusion that stopped by itself. The patient experienced one previous episode of massive transurethral bleeding 2 days before his referral to our institution and was treated with a blood transfusion at another centre. Following spontaneous cessation of transurethral bleeding, the patient was referred to our department for further diagnostic evaluation and treatment. We hospitalized the patient and on the same day of his hospitalization, he experienced a second episode of massive transurethral bleeding. Cystography did not reveal any leakage. Cystoscopy demonstrated no bleeding focus or fistula tract in the neobladder. Abdominal magnetic resonance imaging urography and computerized tomography (CT) did not show any fistula tract between the vascular system and the urinary tract. Angiography showed a small sized pseudo-aneurysm 
of the right external iliac artery and a fistula tract between the pseudoaneurysm and Wallace type ureteral anostomosis (Fig. 1, Fig. 2). Passage of the angiographic contrast material was demonstrated from the right external iliac artery to the ureter. An endovascular stent was inserted at the level of the pseudo-aneurysm and the fistula. Contrast passage ceased following the stent insertion. Therefore, our diagnostic workup and treatment were not delayed due to the unusual nature of this complication. Twelve months after the angiographic stent insertion, our patient did not experience any transurethral bleeding. Currently, he does not have any disease recurrence or progression due to radiologic follow-up.

During the robotic extended pelvic lymph node dissection after the RARC, we skeletonized the vascular structures, including the external iliac arteries, to remove the lymphatic tissue around the vessels that might damage the vascular walls. Additionally, we used monopolar and/or bipolar electrocautery that might cause thermal vascular injury leading to pseudo-aneurysm and fistula development.

\section{Discussion}

RARC with extended pelvic lymph node dissection and extra- or intracorporeal urinary diversion is increasingly being performed.

The most common causes of arterial pseudo-aneurysm development include trauma, tumour, infection, vasculitis, inflammation, chemotherapy, radiotherapy, atherosclerosis, infarction, iatrogenic damage from surgery and angiography. ${ }^{5,6}$ Extra iliac artery pseudo-aneurysm and rupture following renal transplantation and hip prosthesis have also been reported. ${ }^{7,8}$

There is little data on pseudo-aneurysms of the pelvic vessels, particularly following major pelvic surgeries for can-

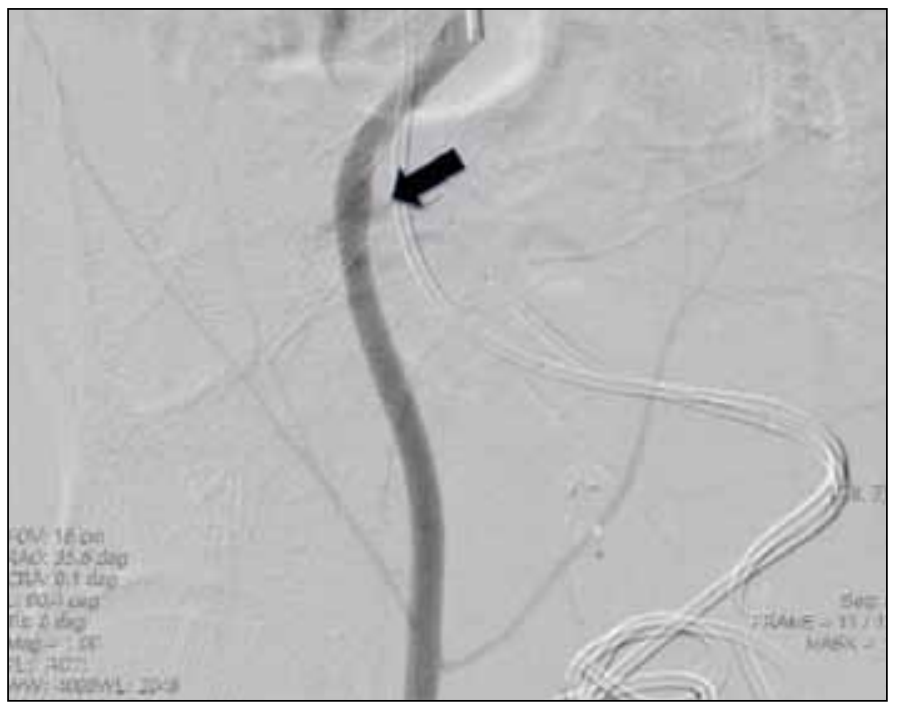

Fig. 1. Angiographic demonstration of right external iliac artery pseudoaneurysm (arrow). cer. The pseudo-aneurysms might develop in the early and late postoperative periods following radical pelvic surgery. Beckley and colleagues reported a case with hemorrhage from an accessory internal pudendal artery pseudo-aneurysm after robotic radical prostatectomy; the patient was successful treated with CT angiography and embolization. This case presented with hematuria on the postoperative day $4 .{ }^{9}$ On the other hand, Ricciardi and colleagues reported a late rupture of external iliac artery pseudo-aneurysm that occurred 2 months after surgery in a patient who underwent pelvic surgery and pelvic lymphadenectomy for cervical cancer. ${ }^{5}$ This case was also successfully treated with endovascular stent placement.

Pseudo-aneurysms are consequences of arterial wall damage. ${ }^{5}$ Our patient did not undergo preoperative or postoperative chemotherapy and/or radiotherapy that might have led to a pseudo-aneurysm. He is a relatively young person for having underlying atherosclerosis; however, he was a heavy smoker and this may have caused a pseudo-aneurysm at the level of the iliac artery. Apart from this, surgery is certainly an important etiologic factor in our case. We watched the recorded video of the whole case and did not detect any suture passage between right external iliac artery and Wallace type ureteral anostomosis (Fig. 3, Fig. 4, Fig. 5, Fig. 6). Arterial wall damage due to monopolar or bipolar electorocautery usage during extended pelvic lymph node dissection around iliac vessels might lead to pseudo-aneurysm development in the late postoperative period. We used large-sized Weck Hem-o-Lok (Teleflex Medical, Research Triangle Park, NC) ligating clips around right external iliac artery during lymph node dissection (Fig. 3). However, we also used monopolar or bipolar electorocautery that might have caused pseudoaneurysm development (Fig. 3). Devascularization injury or thermal vascular injury might be

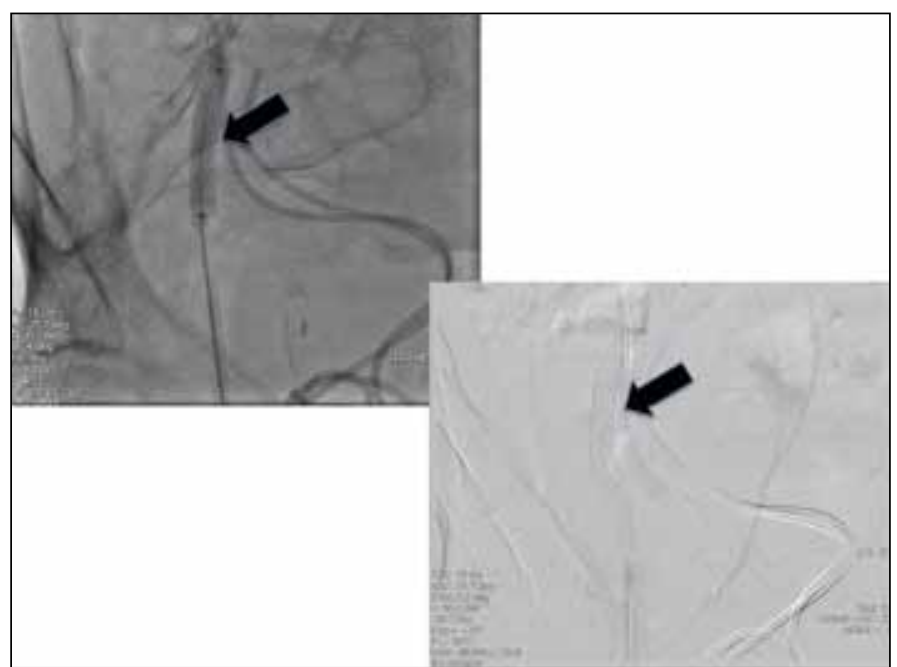

Fig. 2. Endovascular stenting at the level of right external iliac artery pseudoaneurysm and uretero-iliac artery fistula (arrows). 


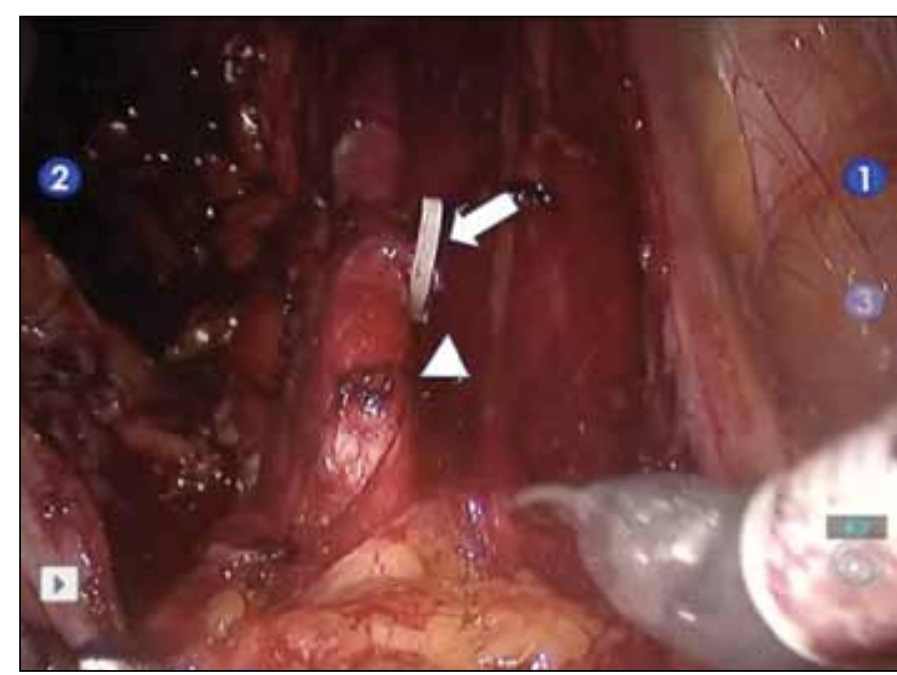

Fig. 3. Appearance of right external iliac artery following robotic extended pelvic lymph node dissection. Arrow: large sized weck Hem-0-Lok ligating clip, Arrowhead: electrocautery site.

factors associated with pseudo-aneurysm and fistula development. In our case, hemorrhage occurred 1 month after the robotic surgery; therefore, a meticulous follow-up is very important in these patients.

To date, we have performed 53 cases of RARC procedures for bladder cancer and we have had only 1 patient who postoperatively developed pseudo-aneurysm of the right external iliac artery - presented in this paper. Regarding the literature on RARC, although vascular injuries have been reported, to the best of our knowledge there is no data related to serious bleeding due to pseudo-aneurysm formation and ureteroiliac artery fistula development postoperatively. However, the real incidence of pseudo-aneurysm formation after a RARC is not known because it cannot be detected due to a lack of complications, such as bleeding.

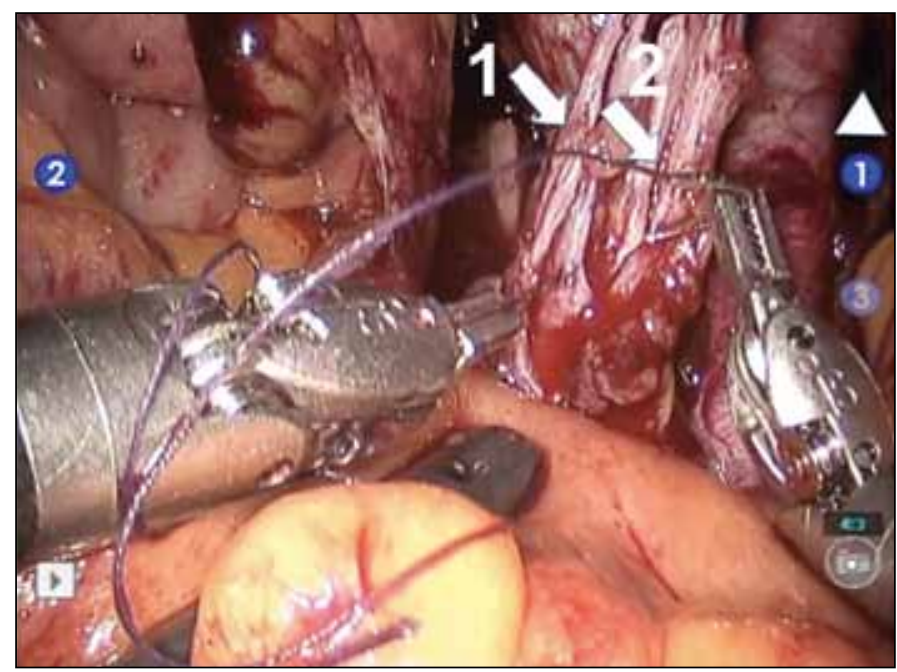

Fig. 5. Appearance of Wallace type uretero-ureteral anostomosis. Arrow-1: right ureter, Arrow-2: left ureter, Arrowhead: right external iliac artery.

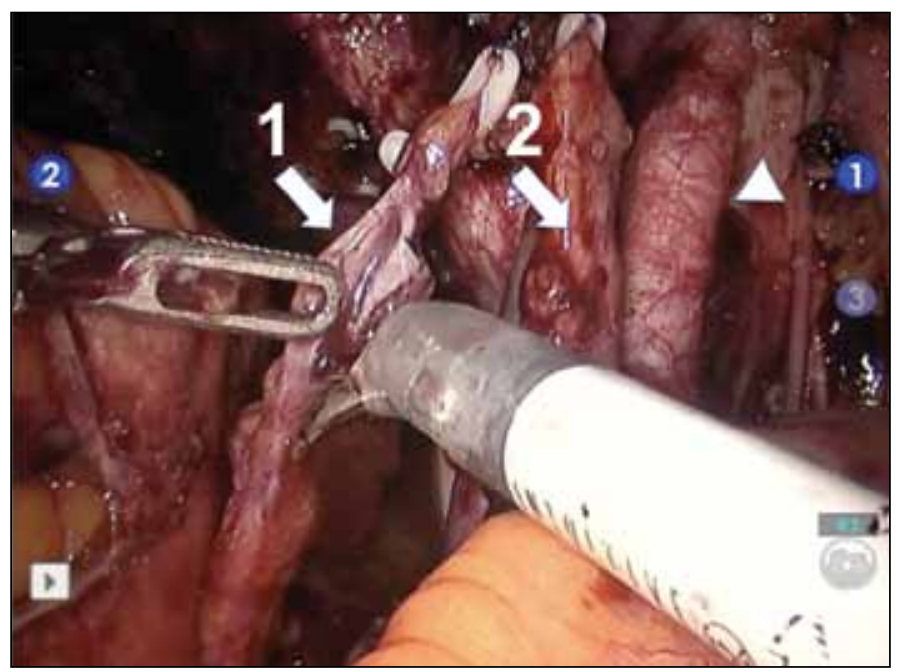

Fig. 4. Spatulation of the right urteter for Wallace type uretero-ureteral anostomosis. Arrow-1: right ureter, Arrow-2: left ureter, Arrowhead: right external iliac artery.

\section{Conclusion}

Angiography and endovascular stenting can be efficiently applied to correctly diagnose and treat massive hemorrhage due to pseudo-aneurysm formation and fistula between the arteries and the urinary tract following RARC. Careful pelvic lymph node dissection, particularly around the vascular structures, and close follow-up in the postoperative period are important in the prevention and awareness of this complication. Massive transurethral bleeding, particularly in the perioperative period, should warn the robotic surgeon on the possible development of a fistula between the arterial system and the urinary tract.

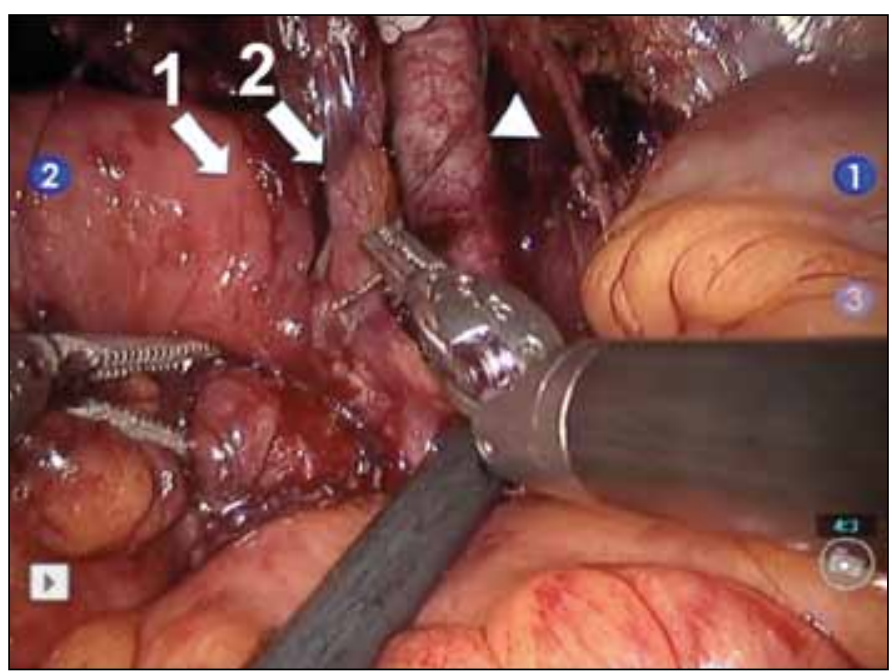

Fig. 6. Appearance of completed Wallace type uretero-ureteral anostomosis and its anostomosis to the chimney of the Studer pouch. Arrow-1: chimney of the Studer pouch, Arrow-2: I Wallace type uretero-ureteral anostomosis, Arrowhead: right external iliac artery. 
Atmaca et al.

Competing interests: None declared.

This paper has been peer-reviewed.

\section{References}

1. Akbulut Z, Canda AE, Ozcan MF, et al. Robot assisted laparoscopic nerve sparing radical cystoprostatectomy with bilateral extended lymph node dissection and intracorporeal Studer pouch construction: Outcomes of first 12 cases. J Endourol 2011;25:1469-79. http://dx.doi.org/10.1089/end.2010.0632

2. Canda AE, Atmaca AF, Altinova $S$, et al. Robot assisted laparoscopic nerve sparing radical cystectomy with bilateral extended lymph node dissection and intracorporeal urinary diversion for bladder cancer: Initial experience in 27 cases. BJU Int 2012;110:434-44. http://dx.doi.org/10.1111/j.1464410X.2011.10794.x

3. Akbulut Z, Canda AE, Atmaca AF, Ozdemir AT, Asil E, Balbay MD. Robot assisted laparoscopic bilatera nerve sparing radical cystoprostatectomy for bladder cancer. J Endourol, Part B, Videourology 2011;25(6).

4. Akbulut Z, Canda AE, Atmaca AF, et al. Robot assisted laparoscopic intracorporeal Studer pouch formation following radical cystoprostatectomy for bladder cancer. J Endourol, Part B, Videourology 2012;26(4).
5. Ricciardi E, Di Martino G, Maniglio P, et al. Life-threatening bleeding affer pelvic lymphadenectomy for cervical cancer: endovascular management of ruptured false aneurysm of the external liliac artery. World J Surg Oncol 2012;10:149. http://dx.doi.org/10.1186/1477-7819-10-149

6. Hampson SJ, Buckenham T, Patel A, et al. False aneurysm of the common iliac artery after pelvic lymphadenectomy: management of an unusual complication. J Urol 1995;154:201-2. http://dx.doi. org/10.1016/S0022-5347(01)67267-7

7. Bracale UM, Carbone F, del Guercio $\mathrm{L}$, et al. External iliac artery pseudoaneurysm complicating renal transplantation. Interact Cardiovasc Thorac Surg 2009;8:654-60. http://dx.doi.org/10.1510/ icvts.2008.200386

8. Smith GH, Nutton RW, Fraser SC. lliac artery pseudoaneurysm rupture following excision of an infected hip prosthesis. J Arthroplasty 2011;26:977.e13-5.

9. Beckley I, Patterson B, Hamaday M, et al. Case report: delayed hemorrhage from an accessory internal pudendal artery pseudoaneurysm after robotic radical prostatectomy: successful management with ct angiography and embolization. J Endourol 2007;21:923-5. http://dx.doi.org/10.1089/end.2006.0419

Correspondence: Dr. Abdullah Erdem Canda, Associate Professor of Urology, Yildirim Beyazit University, School of Medicine, Ankara Ataturk Training \& Research Hospital, Department of Urology, Bilkent, Ankara 06800 Turkey; erdemcanda@yahoo.com 